\title{
Efectividad de
}

las estrategias

de enseñanza

implementadas

en la asignatura

de Seminario de

Formación Integral.

MSc. Alvaro Antonio Muñoz González 


\section{Efectividad de las estrategias de enseñanza implementadas en la asignatura de Seminario de Formación Integral}

Copyright $@ 2018$ UNAN-Managua Todos los DerechosReservados.

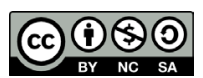

MSc. Álvaro Antonio Muñoz González. Docente del Departamento de Psicología, UNAN -Managua.

ID Orcid: https://orcid.org/ 0000-0002-6688-6102 amunoz@unan.edu.ni

Recibido: 20/06/2018 Aceptado: 15/08/2018

\section{Effectiveness of the teaching strategies implemented in the course of the Integral Education Seminar}

\section{RESUMEN}

La participación activa y beligerante de los docentes universitarios en la implementación de estrategias de enseñanza juega un papel importante en la inclusión y motivación de los estudiantes por su desarrollo personal. En las carreras de la Facultad de Humanidades y Ciencias Jurídicas, donde se imparte la asignatura de Seminario de Formación Integral, se han implementado diversas estrategias de enseñanza, por ello este artículo ${ }^{1}$ analiza la efectividad de las mismas; La metodología empleada está basada en un enfoque cuantitativo y cualitativo, con un diseño no experimental descriptivo de corte trasversal. Entre los resultados encontrados con una muestra de 13 docentes seleccionados y 280 estudiantes de las carreras de esta facultad, destaca que la actitud de los docentes difiere en cada uno de los grupos seleccionados en esta investigación, ya que, a pesar de trabajar con planificaciones y estrategias didácticas homologadas, derivadas del trabajo de colectivos docentes, cada docente imprime un toque particular a su trabajo cotidiano y esto impacta en la efectividad de las estrategias. Se determina que las técnicas empleadas por los docentes a la hora de impartir la clase de Seminario de Formación Integral lograron alcanzar una efectividad limitada, puesto que no todos los estudiantes las calificaron como buenas, motivadoras, ajustadas a la realidad, expectativas de formación y utilidad profesional.

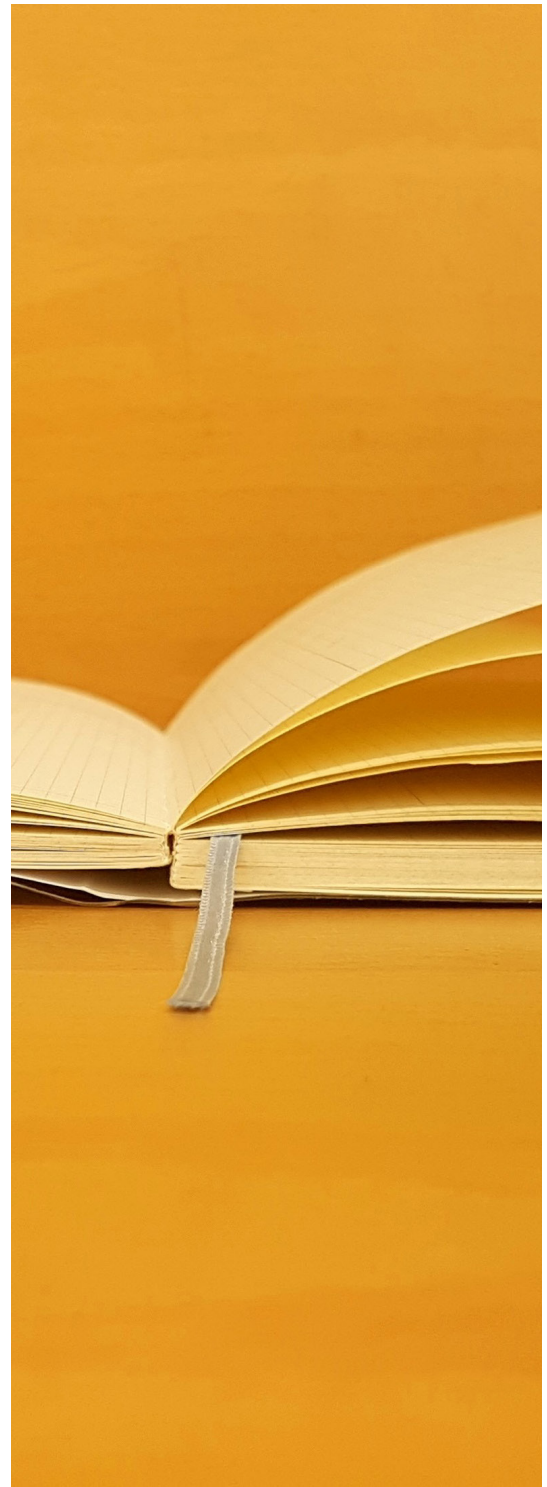

\section{PALABRAS CLAVE}

Estrategias, enseñanza- aprendizaje, efectividad, estudiantes, docentes.

\footnotetext{
1 El presente artículo, es el resultado de la ejecución de procesos investigativos, generados a partir de los fondos concursables para la investigación otorgados por el vicerrectorado de Investigación, Postgrado y Extensión Universitaria de la UNAN-Managua a docentes y estudiantes universitarios en el marco del fortalecimiento de las capacidades y experticia investigativa
} 


\section{ABSTRACT}

The active and belligerent participation of university teachers in the implementation of teaching strategies plays an important role in the inclusion and motivation of students. In the careers of the Faculty of Humanities and Legal Sciences, where the subject of Integral Training Seminar is taught, various teaching strategies have been implemented, so this article analyzes their effectiveness. The methodology used is based on a quantitative and qualitative approach, with a descriptive non-experimental design of transversal cut. Among the results found with a sample of 13 selected teachers and 280 students of the careers of this faculty, it stands out that the attitude of teachers differs in each of the groups selected in this research, since, despite working with schedules and Approved teaching strategies, derived from the work of teaching groups, each teacher prints a particular touch to their daily work and this impacts the effectiveness of the strategies. It is determined that the techniques used by teachers when teaching the Integral Training Seminar class achieved limited effectiveness, since not all students rated them as good, motivating, adjusted to reality, expectations of training and usefulness professional.

\section{KEYWORDS}

Strategies, teaching-learning, effectiveness, students, teachers.

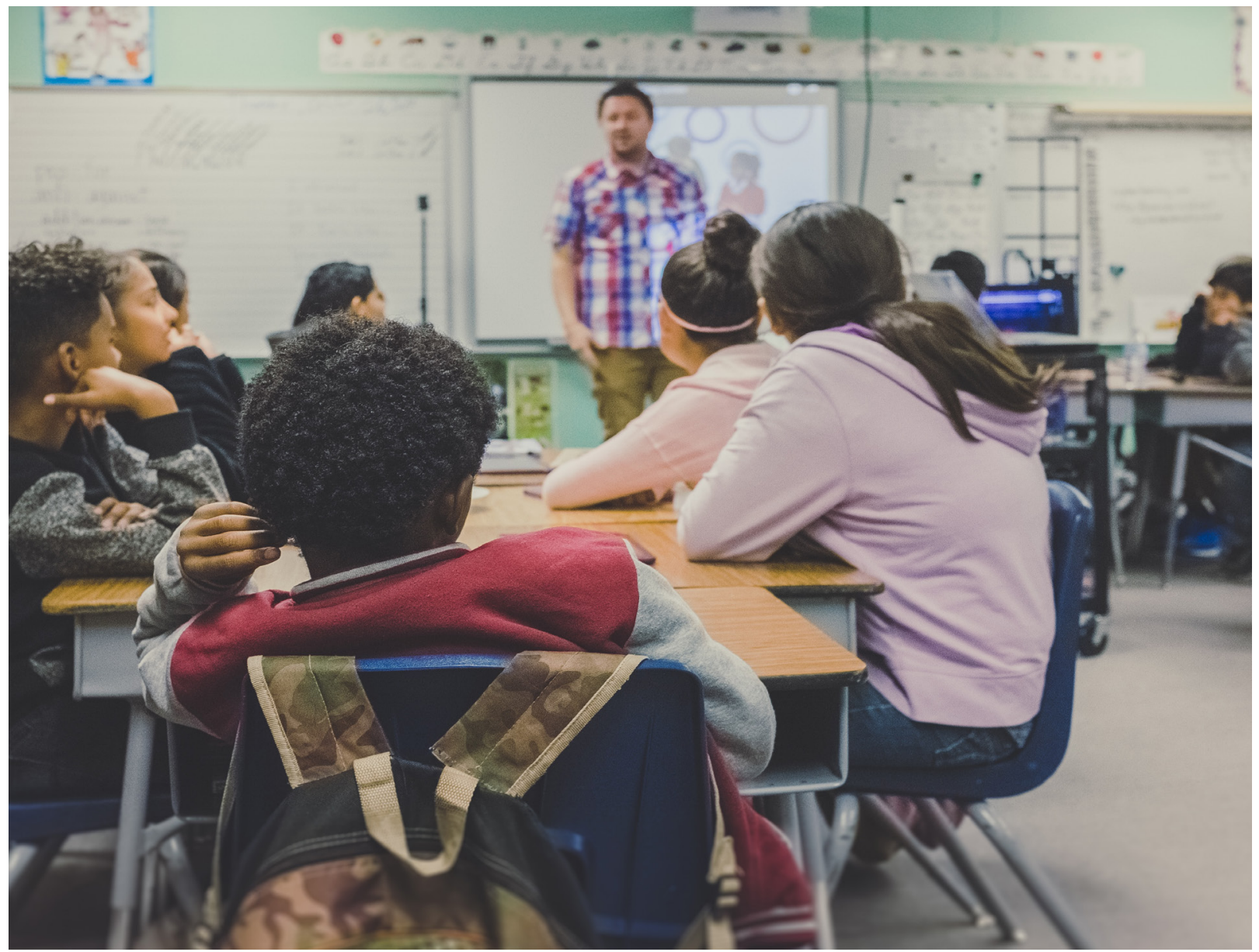




\section{Introducción}

El modelo Educativo de la UNAN-Managua, contempla la formación de profesionales con compromiso científico y humanista hacia el mundo que les rodea, con un sentido crítico, reflexivo y propositivo, a fin de que contribuyan a la solución de problemas de sus entornos reales. En esta vía, podemos entender que la selección e implementación de estrategias de enseñanza que favorecen el aprendizaje, debe partir de una concepción en la que el sujeto es el centro del modelo y es un ente activo y regulador de sus propios procesos para la correcta aplicación de lo aprendido.

Como parte de la malla de formación común a todas las carreras ofertadas por la UNANManagua, se encuentra la asignatura Seminario de Formación Integral, misma que se imparte entre el segundo y tercer semestre a todos los estudiantes de la universidad, y que comprende una serie de temáticas básicas, presentadas de forma multidisciplinaria en los contenidos de: Derechos Humanos, Género, Desarrollo Humano Sostenible y Soberanía y Seguridad Alimentaria y Nutricional; se pretende que en esta asignatura el estudiante fortalezca y desarrolle los conocimientos y actitudes, prácticas que estimulen una ética de vida y contribuyan al desarrollo sostenible de la sociedad nicaragüense.

Esta área común de la Formación Integral de los estudiantes universitarios se enfrenta a dificultades, principalmente el aspecto motivacional de los discentes, y en ocasiones incluso, la aplicación de inadecuadas estrategias didácticas por parte del docente, mismas que no Ilenan las expectativas de los estudiantes ni vinculan lo abordado con la realidad social ni con el campo profesional en formación. El estudio se centra en identificar las principales estrategias de enseñanza implementadas en la asignatura por parte de los docentes y determinar la incidencia de las mismas, partiendo de la valoración de los estudiantes en el desarrollo de la clase.

\section{Material y métodos}

El foco de investigación del presente estudio, está centrado en las Estrategias de enseñanza implementadas en la asignatura de Seminario de Formación Integral en las Carreras de la Facultad de Humanidades y Ciencias Jurídicas de la UNAN-Managua. Dado que, el estudio implica una valoración por parte de los estudiantes de la efectividad de las estrategias en su aprendizaje, se parte de la utilización de un paradigma de investigación mixto, el cual tiene como característica que la recolección y análisis de información se realizan mediante datos cuantitativos y cualitativos para llegar al establecimiento de meta inferencias más allá de las estadísticas y más allá de las categorías cuantitativas.

El estudio es de corte transversal, ya que se realiza con estudiantes que cursaron la asignatura en el año lectivo 2017, se aplicó un muestreo aleatorio estratificado con todos los grupos, partiendo de una muestra de 280 estudiantes de los 13 grupos estudiados. La información fue obtenida principalmente mediante la observación directa en el aula de clases y la aplicación de instrumento Escala tipo Likert (estructurado en 30 Ítems, con tres categorías que son: Estrategias de enseñanza, valoración del vínculo de la asignatura con el desempeño profesional presente y futuro y desempeño docente), mismo que fue sometido a un proceso de validación de contenido, tanto por criterio de expertos, como por coeficiente de consistencia interna de los ítems, obteniendo un coeficiente de Alfa de Crombach de 0.985 . 
Posterior a la aplicación de los instrumentos de tipo escala, los datos obtenidos fueron procesados a través del Programa Estadístico para las Ciencias Sociales (SPSS por sus siglas en ingles) en su versión 23, lo que permitió la creación de categorías para la facilitación de la interpretación a un nivel descriptivo de los resultados y la explicación con fundamentos teóricos de los hallazgos.

\section{Resultados y discusión}

Es importante para alcanzar los objetivos planteados al inicio de esta investigación, destacar que una estrategia didáctica se define como un sistema de planificación que se aplica a un conjunto de acciones que tienen por finalidad alcanzar una meta educativa concreta. Por lo tanto, no podemos hablar de implementación de estrategias si no hay una meta que perseguir por parte del docente y la institución educativa en sí. Es importante dentro del proceso de enseñanza- aprendizaje que el docente planifique de manera consciente y reflexiva, las técnicas y actividades que puede utilizar, a fin de que ese alcance de los objetivos de aprendizaje, logre la construcción y significatividad del mismo, en conjunto con los estudiantes.

El concepto de estrategia didáctica, responde entonces, en un sentido estricto, a un procedimiento organizado, formalizado y orientado para la obtención de una meta claramente establecida. Su aplicación en la práctica requiere del perfeccionamiento de procedimientos y de técnicas cuya elección detallada y diseño son responsabilidad del docente, es por eso que Silvia. R. (2009) plantea que para definir cuáles serán las estrategias de enseñanza más adecuadas, es necesario tomar en cuenta los contenidos disciplinares y de las estrategias para presentarlos a los alumnos, pero más aún reflexionar sobre las características particulares de los estudiantes.

Con este estudio se pudo determinar cuáles son esas estrategias de enseñanzas que implementan los docentes a la hora de impartir la clase de Seminario de Formación Integral. Por medio de los instrumentos de investigación, se logró identificar que existen 11 tipos de estrategias utilizadas por los maestros para captar la atención del alumnado, entre las cuáles resaltan el seguimiento o secuencia lógica de los contenidos, así como la presentación novedosa de los mismos, el esfuerzo del docente por vincular conocimientos previos con los nuevos adquiridos, la realización de simulaciones pedagógicas o sociodrama para ejemplificar las problemáticas abordadas en la clase, la técnica de lluvia de ideas, preguntas para explorar los conocimientos previos sobre el tema a abordar, realización de resúmenes tomando en cuenta la participación del estudiando, utilización de ilustraciones para la comprensión de las temáticas, la facilitación de guías metodológicas, y el reconocimiento público de los aportes valiosos de otras personas para facilitar la clase. 
Uno de los aspectos que llama la atención de la valoración de los estudiantes, radica principalmente en la forma en que se presentan por parte de los docentes, todos los contenidos que se desarrollan a lo largo de la asignatura. En el gráfico que se presenta a continuación, podemos ver que, en líneas generales, los estudiantes de la Facultad de Humanidades y Ciencias Jurídicas, consideran que los contenidos son presentados por parte de los docentes de manera novedosa, interesante y eficaz. En un $49 \%$ de las veces, los estudiantes se mostraron muy de acuerdo con el enunciado, $32 \%$ del estudiantado está de acuerdo, $10 \%$ se muestra indiferente no estando ni de acuerdo ni en desacuerdo, por otro lado, si se suma las opiniones desfavorables con respecto a la manera en que se presentan los contenidos, encontraremos que solamente un $9 \%$ de los estudiantes, no consideran que los contenidos de la asignatura sean interesantes en la manera en que se presentan, esto contra un $81 \%$ de valoración positiva del estudiantado.

\section{Gráfica 1. Los contenidos de la asignatura son presentados de manera novedosa, interesante y eficaz.}

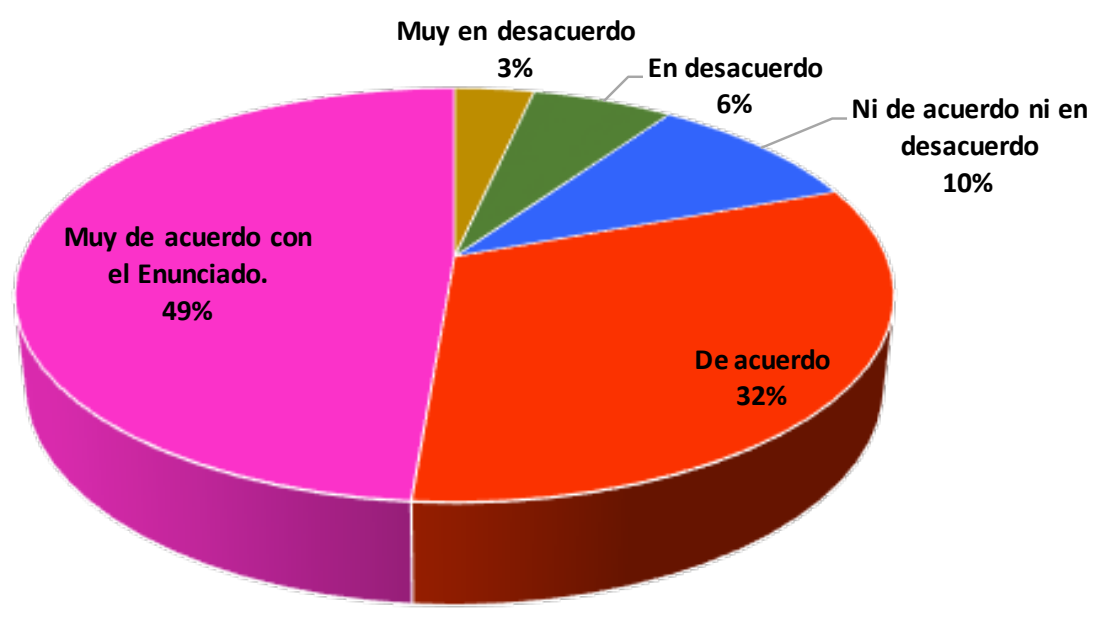

Fuente: Elaboración del autor

Otro aspecto importante a destacar, es la manera en la que el docente facilita la clase y si esto despierta el interés por los temas que anteriormente los estudiantes consideraban insignificantes o pasaban desapercibidos en su vida diaria. En este sentido nos encontramos que $48 \%$ de los estudiantes valoran de manera significativamente positiva la manera en la que el docente facilita la clase aunado a un $38 \%$ que se presenta de acuerdo con respecto al enunciado, nos encontramos con qué el $86 \%$ de estudiantes valoran positivamente este aspecto de la función docente. Solamente un $5 \%$ de los estudiantes, se muestra indiferente no estando ni de acuerdo ni en desacuerdo, repitiéndose el hecho de que un $9 \%$ de los estudiantes tienen una valoración negativa de la manera en la que el docente facilita la clase; dividiéndose esto en un $6 \%$ que se muestra en desacuerdo con el enunciado, y $3 \%$ muy en desacuerdo con el mismo. 


\section{Gráfica 2. La manera en que el docente facilitó la clase despertó mi interés por temas que anteriormente pasaban desapercibidos en la vida diaria.}

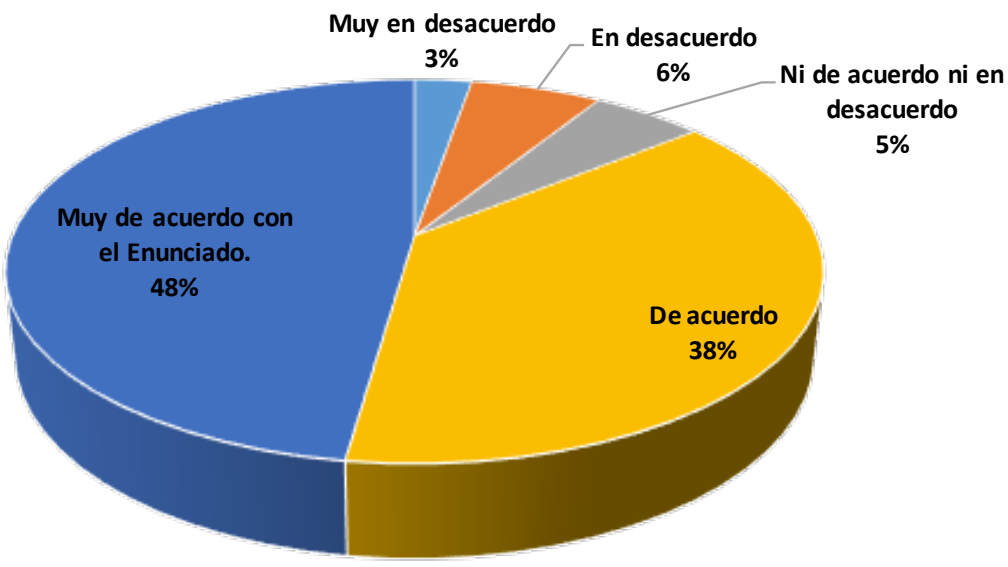

Fuente: Elaboración del autor

En cuanto al acápite de si los temas abordados en cada una de las clases activaron la curiosidad de los estudiantes para aprender al respecto, encontramos una valoración aún más favorable, ya que si sumamos el $53 \%$ de estudiantes que se muestran muy de acuerdo con el enunciado con el $35 \%$ que se describen de acuerdo, concluiremos que el $88 \%$ de los estudiantes valoran que los temas desarrollados a lo largo de la asignatura son interesantes, y les motivan a la búsqueda de mayor cantidad de información respecto a los fenómenos estudiados.

En este acápite, tanto los estudiantes que se muestran indiferentes, como aquellos que valoran de manera negativa el ítem disminuye, teniendo solamente $6 \%$ de estudiantes que no se me describe de acuerdo ni en desacuerdo, $4 \%$ de ellos en desacuerdo y $2 \%$ muy en desacuerdo lo que suma para una valoración desfavorable de apenas $6 \%$ del estudiantado.

Gráfica 3. Los temas abordados en las clases fueron presentados de forma que activaron mi curiosidad e interés por aprender más al respecto.

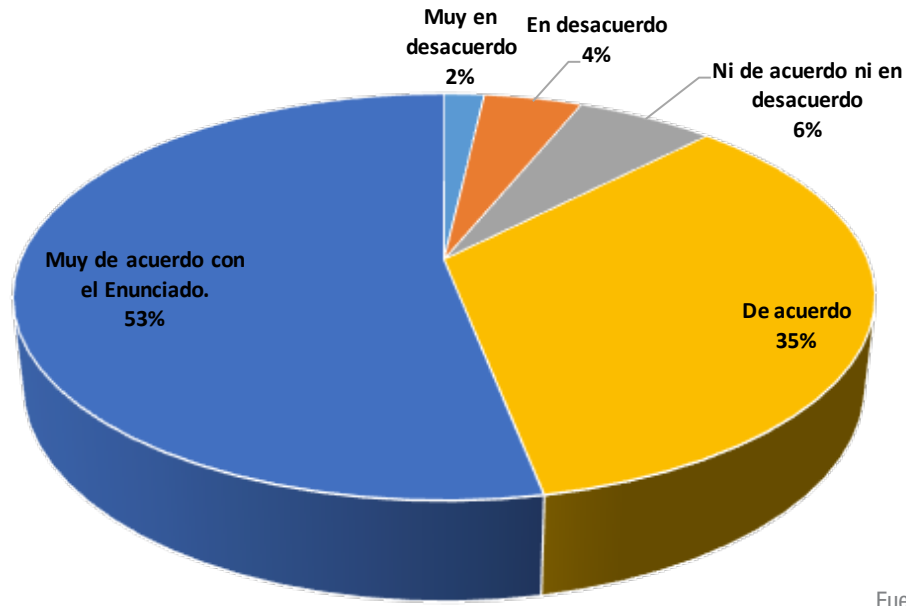


Al momento de valorar si los aprendizajes adquiridos serán de utilidad en su futuro profesional, refleja que $87 \%$ de los estudiantes concuerdan con el enunciado dividiéndose está en un $65 \%$ que está muy de acuerdo y un $20 \%$ que se encuentran acuerdo con el mismo, $5 \%$ de los estudiantes se muestran ni de acuerdo ni en desacuerdo $4 \%$ en desacuerdo y $4 \%$ muy en desacuerdo con el enunciado.

Esto implica, que en su desarollo temático la asignatura contempla contenidos que son de mucha relevancia para los estudiantes de la facultad, y que los mismos son vinculados con el quehacer profesional de cada una de las especialidades que se imparten en la misma.

Lo que guarda una estrecha relación con el gráfico número 5 , que describe el sentimiento que tienen los estudiantes con respecto a los saberes necesarios en el futuro ejercicio de su profesión y los contenidos de la asignatura, en este sentido encontramos que $91 \%$ de los estudiantes están de acuerdo dividiéndose esto en $70 \%$ muy de acuerdo $21 \%$ de estudiantes de acuerdo $4 \%$ ni de acuerdo ni en desacuerdo, $3 \%$ el desacuerdo y $2 \%$ muy en desacuerdo con el enunciado.

Cuando se valora las estrategias didácticas usadas en la clase es importante destacar que en un $88 \%$ estas son valoradas de manera favorable, $64 \%$ de los estudiantes consideran que estas estrategias son muy buenas $24 \%$ las valoran como muy buenas, solamente $9 \%$ de los estudiantes consideran que las estrategias cumplen regularmente su función y $3 \%$ que emiten como criterio de juicio, que las estrategias no son las adecuadas, categorizándolas como malas, esto, según se pudo constatar en el aula de clase, tiene mucho que ver con los horarios y las condiciones de infraestructura y medios tecnológicos con los que se desarrolla la docencia, ya que las estrategias y temas a desarrollar son homologados en colectivo docente.
Gráfica 4: Considero que he adquirido aprendizajes que me serán de mucha utilidad en mi futuro ejercicio profesional.

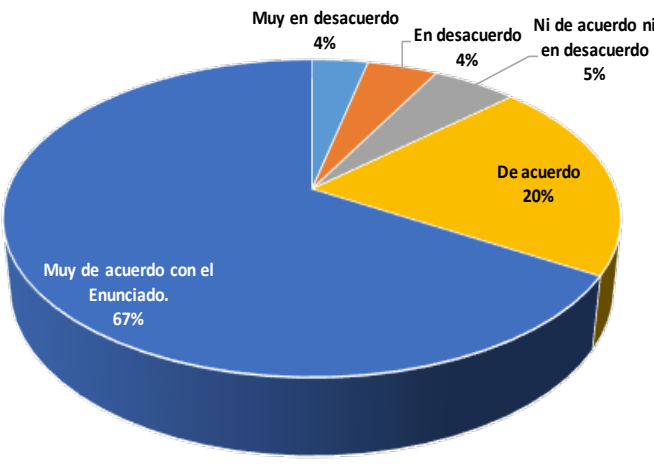

Fuente: Elaboración del autor

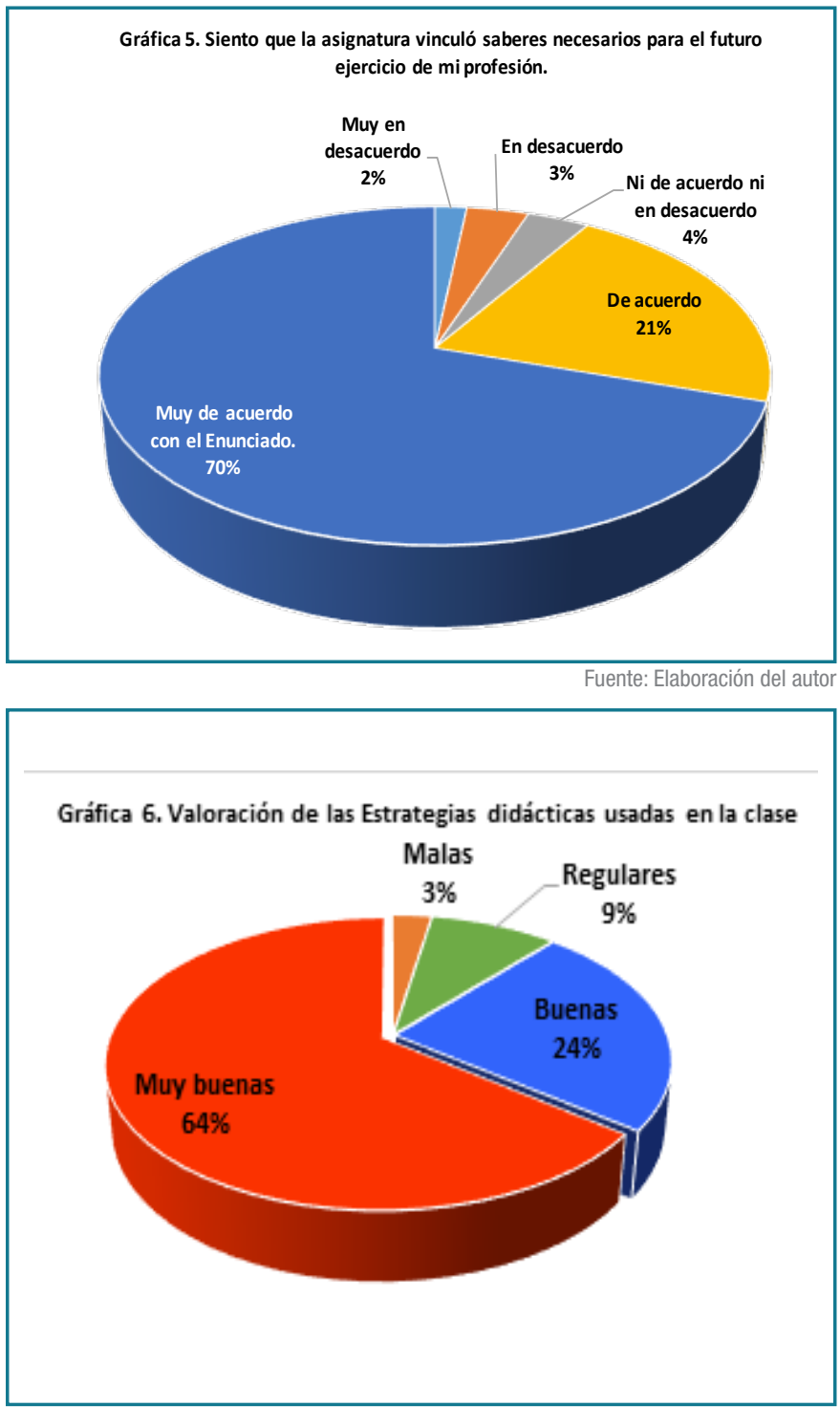

Fuente: Elaboración del autor 
La actitud que los estudiantes tienen para con la asignatura y sus alcances despunta con que $8 \%$ de los estudiantes tienen una valoración negativa de la asignatura, 2\% muy desfavorable y $6 \%$ desfavorable, un $20 \%$ aceptable y un $72 \%$ tiene una actitud muy favorable para con la asignatura cursada.

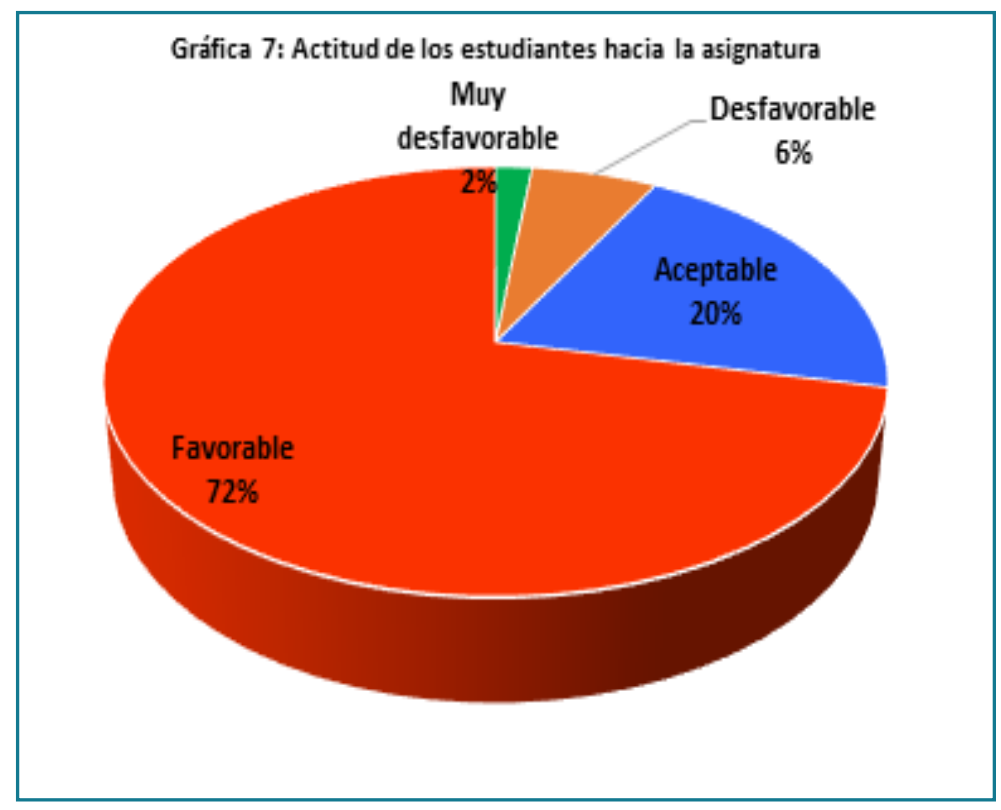

Fuente: Elaboración del autor

\section{Gráfica 8:Valoración de la labor docente por carrera}

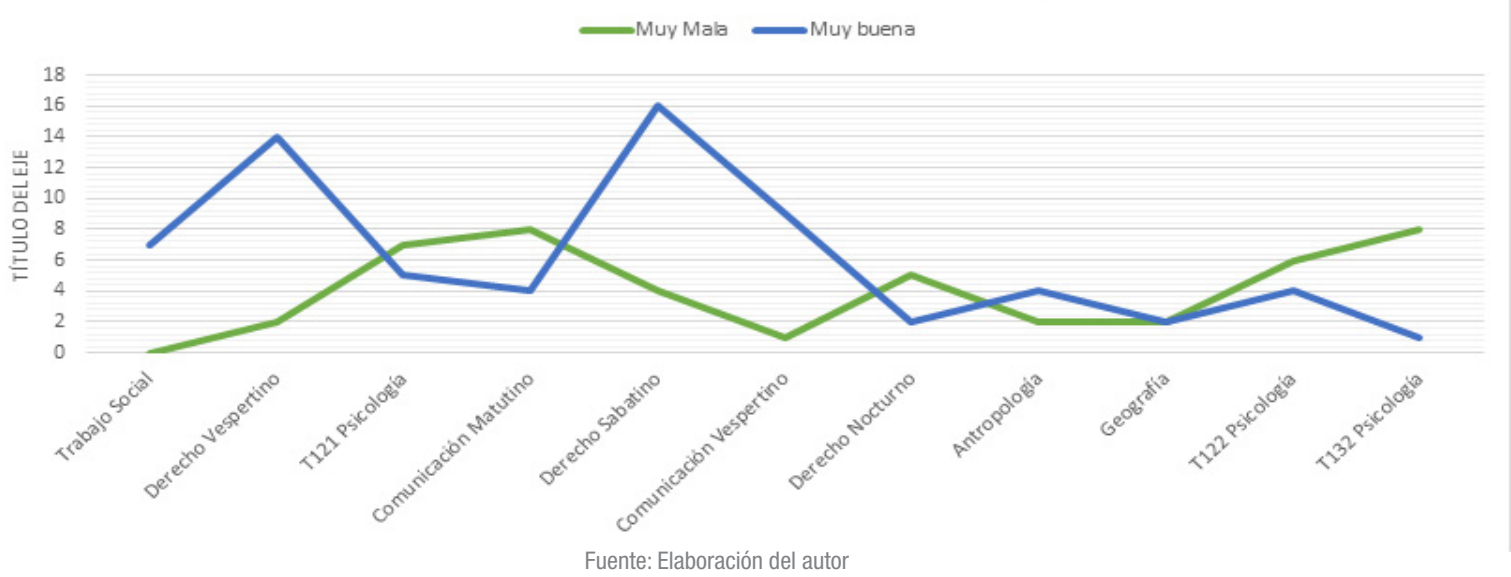

El gráfico que condensa la valoración que tienen los estudiantes con respecto a la labor del docente en cada una de las carreras refleja una polaridad en este aspecto, ya que no existen posiciones intermedias, los estudiantes valoran esta labor como muy buena o muy mala y podemos observar cada una de las carreras de la Facultad con la valoración que los estudiantes tienen del docente y su labor al momento de impartir la asignatura, destacando en esta la valoración positiva que tienen los estudiantes de la carrera de Derecho en algunas modalidades, y la valoración desfavorable que tienen algunos estudiantes en carreras como Psicología, Derecho en su modalidad nocturna y la carrera de Diplomacia y Ciencias Políticas. 


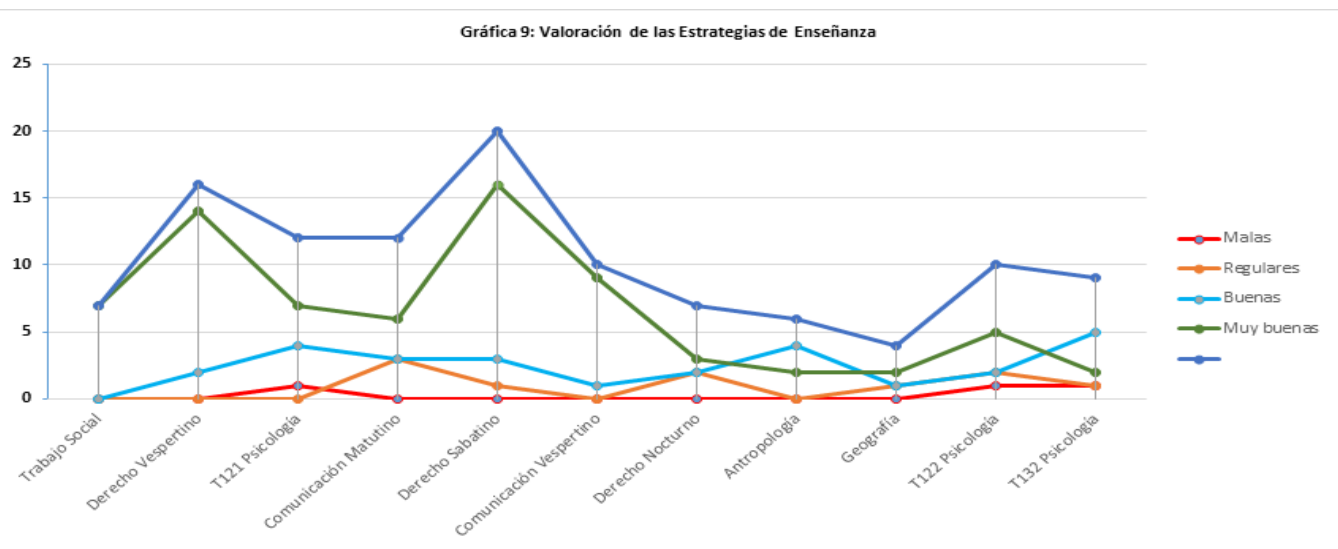

Fuente: Elaboración del autor

Un comportamiento similar encontramos al evaluar las estrategias de enseñanza por parte de los estudiantes. En este sentido encontramos que, normalmente, en cada una de las carreras hay una valoración EXCELENTE de las estrategias, esto por encima de la valoración de MUY BUENAS, BUENAS, REGULARES Y MALAS. Solamente la carrera de Psicologia, realiza una valoración sobre las estrategias didácticas de MALAS.

\section{Conclusiones}

Para concluir, podemos puntualizar que por parte de los estudiantes de la Facultad de Humanidades y Ciencias Jurídicas de la UNAN-Managua existe una valoración favorable de los contenidos y de la forma en la que son presentados los mismos en la asignatura de Seminario de Formación Integral. Esto está muy vinculado con la valoración que los estudiantes poseen de aspectos como: la manera en que el docente facilita la asignatura, los temas abordados en la clase en términos de interés y curiosidad generado por parte del estudiantado, los aprendizajes adquiridos a lo largo de la asignatura, el vínculo que estos temas tiene con la realidad que viven los estudiantes, con el perfil de la profesión que el estudiante pretende ejercer y con las estrategias didácticas usadas en la clase.

Los estudiantes muestran una actitud sumamente favorable para con la asignatura, de igual manera, con la labor docente exceptuándose muy pocas carreras y muy pocas modalidades, al respecto de las estrategias de enseñanza, cabe destacar que el comportamiento normal de este acápite es que los estudiantes en todas las carreras de la Facultad de Humanidades y Ciencias Jurídicas las valoran como EXCELENTES por encima de otros constructos de valoración, se encontró que en muy pocas carreras y en muy pocas modalidades existe una valoración desfavorable, considerando como REGULARES o MALAS las estrategias de enseñanza.

Resulta un tema importante a destacar la importancia que posee en este entorno de enseñanzaaprendizaje la implementación real por parte de los docentes de estrategias que resulten innovadoras y aterrizadas a la realidad del país y los educandos, tema de vital importancia para la consecución de la educación de calidad en el siglo XXI. 


\section{Referecias bibliográficas}

- $\quad$ Anijovich. Rebeca \& Mora. Silvia. (2009). Estrategias de enseñanza, otra mirada al quehacer en el aula. [Fecha de consulta: 26 de enero de 2018] Disponible en: http://www.terras.edu.ar/ biblioteca/3/3Como-ensenamos-Las-estrategias-entre-la-teoria-y-la-practica.pdf

- Carles Monereo (coord.), Montserrat Castelló, Mercé Clariana, Montserrat Palma, María L. Pérez. (1999) Estrategias de enseñanza y aprendizaje Formación del profesorado y aplicación en la escuela. 185 páginas.

- Elnia Libeth Fuentes Castillo, María Inés Blandino. Efectividad de las estrategias didácticas que implementan los docentes en la formación de estudiantes, curso de profesionalización. 13 páginas.

- Iranzu, Soto Ortigosa. (2012) Análisis de la efectividad de las estrategias didácticas motivacionales aplicadas a una unidad didáctica. Proyecto fin de máster Formador de Profesorado de educación Secundaria. 38 páginas.

- Universia Argentina (2017). 6 estrategias para promover la participación y el compromiso de los estudiantes en clase). [fecha de consulta: 11 de octubre de 2018]. Recuperadode:http:// noticias.universia.com.ar/educacion/noticia/2016/08/12/1142674/6-estrategias-promoverparticipacion-compromiso-estudiantes-clase.html.

- Valle Arias, Antonio, Barca Lozano, Alfonso, González Cabanach, Ramón, Núñez Pérez, José Carlos, Las estrategias de aprendizaje revisión teórica y conceptual. Revista Latinoamericana de Psicología [en línea] 1999, 31 [Fecha de consulta: 18 de septiembre de 2018] Disponible en:<http://www.redalyc.org/articulo.oa?id=80531302> ISSN 0120-0534 